\title{
1980. L’encyclopédie en colonne
}

\section{Nicolas Rieder}

\section{(2) OpenEdition}

Journals

Édition électronique

URL : http://journals.openedition.org/edl/2409

DOI : 10.4000/edl.2409

ISSN : 2296-5084

\section{Éditeur}

Université de Lausanne

\section{Édition imprimée}

Date de publication : 15 mars 2020

Pagination : 159-162

ISBN : 978-2-940331-73-4

ISSN : 0014-2026

\section{Référence électronique}

Nicolas Rieder, « 1980. L'encyclopédie en colonne », Études de lettres [En ligne], 312 | 2020, mis en ligne le 24 mars 2020, consulté le 17 septembre 2020. URL : http://journals.openedition.org/edl/2409

DOI : https://doi.org/10.4000/edl.2409

(c) Études de lettres 


\section{L'ENCYCLOPÉDIE EN COLONNE}

«Ce n'est pas parce qu'il y a chiottes dans Le petit Robert ou gogues qu'il est meilleur." Alain Rey n'a pas attendu de participer à un morceau de rap en 2007 pour témoigner d'une certaine truculence, puisque ces paroles sont extraites du $249^{\mathrm{e}}$ numéro d'Apostrophes, diffusé le 12 septembre 1980. Le magazine de Bernard Pivot était consacré en cette occasion à «la Guerre des dictionnaires", expression dont on userait plus spontanément pour qualifier, au XIX ${ }^{e}$ siècle, la lutte entre Émile Littré et Pierre Larousse. Il faut dire que les quelque 1'200'000 dictionnaires vendus chaque année en France, en ce temps où le secteur scolaire/ encyclopédie mangeait son pain blanc, attisaient les convoitises.

L'entourloupe de Gérard Pirès, sorti en avril 1980, met d'ailleurs en scène des escrocs à la petite semaine, devenus colporteurs d'encyclopédies médicales dans les marais poitevins. La devise de Jean-Pierre Marielle, qui joue le rôle du chef de vente? "Le plaisir d'un vrai vendeur c'est de vendre à des gens qui n'ont absolument pas besoin de ce qu'on leur propose ou qui n'ont pas de quoi se le payer.» Un peu plus d'un siècle après l'invention de la vente à tempérament, dont le modèle économique a permis l'essor des ouvrages de Littré et Larousse, la vente à crédit sert de ressort comique à un film où paysans crédules et vendeurs cabotins se livrent bataille. Aux hâbleurs la première manche, qui voit une mère de famille nombreuse se lamenter devant le garde-manger vide; aux paysans la revanche, qui caillassent les aigrefins et brûlent l'encyclopédie. Discutable sur le contenu, pas même sauvé par les dialogues d'Audiard ou le jeu de Dutronc, L'entourloupe véhicule l'image séculaire du colporteur malhonnête à un moment où le modèle économique de l'édition va être profondément bouleversé. 
Ni boue ni patois en effet dans les salons privés de Matra, où l'on situerait volontiers la préparation de l'OPA que Jean-Luc Lagardère va lancer sur Hachette en décembre 1980. Cette prise de contrôle ouvre la voie aux holdings qui déboucheront sur la situation actuelle, où trois mastodontes dominent le champ éditorial français. Or, au terme d'un incessant ballet d'acquisitions, cessations, rachats, Lagardère (et ses différents avatars) a possédé à un moment de ces quarante dernières années les cinq maisons (Larousse, Le Robert, Bordas, Hachette et Flammarion) qui étaient présentes sur le plateau d'Apostrophes pour défendre leur dictionnaire respectif. Retour sur Antenne 2 donc, où Bernard Pivot n'aura jamais si bien porté son nom, lui qui a organisé deux rangées de belligérants rebaptisés pour l'occasion. À sa droite, les tenants du haut du pavé: M. Larousse (Claude Dubois), M. Robert (Alain Rey) et M. Logos (Jean Giraudet); à sa gauche, les prétendants: $M$. Hachette (Vincent Brugère) et M. Quillet Flammarion (Jean-Marie Pruvost-Beaurain).

"Le monde a changé, le petit Larousse aussi»; «Le monde a changé, le dictionnaire aussi» (Hachette) ; "Plus pratique, plus précis, plus moderne. Le Petit Flammarion.» Sur le plateau, chacun a souci d'illustrer sa campagne publicitaire, tentant de se démarquer, de se singulariser. M. Larousse défend la disparition d'écrivains comme Pierre Benoît ou Henry Bordeaux par le fait qu'ils étaient caractéristiques de leur époque, mais pas de 1980, et qu'il faut laisser la place au jazz ou à la bande dessinée. M. Quillet Flammarion prend une posture plus progressiste encore; on oublie le «jojo" d'« affreux jojo»; on accueille «jojoba», que personne ne connaît:

si cette huile [tirée du jojoba] pouvait rendre les mêmes services que l'huile de cachalot, peut-être que les cachalots seraient moins chassés. D’un point de vue écologique, cela peut être un argument intéressant.

Machines à sous, les dictionnaires et les encyclopédies témoignent également des idéologies qui sous-tendent leur programme; ils n'en sont pas moins également des "machines à rêver", comme le disait Roland Barthes en 1980.

Nous n'avons pas encore évoqué le sixième invité de l'émission qui n'est ni lexicographe, ni responsable d'édition, mais écrivain: il s'agit de Jean-Luc Benoziglio qui vient de faire paraître Cabinet portrait. Le narrateur de ce roman emménage dans un appartement trop exigu pour contenir sa bibliothèque, et son Encyclopaedia Britannica en 
25 volumes se retrouve dans les toilettes de l'étage. Pas encore en ligne, l'encyclopédie est en colonne, sous le lavabo, et les cabinets d'aisance deviennent cabinet d'études, par un renversement rabelaisien. Mais là où Ponocrates s'enfermait avec le seul Gargantua pour lui expliquer «les points les plus obscurs et difficiles» des saintes Écritures, le recours aux cabinets communs permet ici une réflexion sur la démocratisation du savoir: la concierge de l'immeuble se fiche de l'encyclopédie, faute d'y trouver "Guytrone et Zilux»; Sébastien, un jeune autiste, en arrache les planches anatomiques; quant au voisin de palier, il finit par faire des pages un usage plus conforme à ce qu'on attendrait du papier dans un tel lieu. Loin de faire la leçon, Benoziglio questionne la hiérarchie des savoirs (culture populaire/culture érudite), le médium de leur diffusion (télévision/livre) et la relation que chacun peut entretenir avec les connaissances.

On devine assez clairement l'auteur sous les traits du narrateur. Le père de Benoziglio était né en Turquie et arrivé en Suisse en 1920 pour y étudier la médecine. À sa mort, le romancier découvre la judéité de celui qu'il nomme "l'homme en blouse blanche». Dans Cabinet portrait, la quête des origines passe par des articles de l'encyclopédie qui portent sur la ville d'enfance du père, le judaïsme, etc. Ce que Bernard Pivot résume parfaitement: "[le narrateur] recherche les marques d'un destin individuel dans un ouvrage collectif." Mais "c'est parfaitement dérisoire", lui répond l'auteur, en écho à son roman: «mais entre Benoni [...] et Bensberg [...], il n’y avait rien. / Ni personne. / On s'en serait douté.» ${ }^{1}$ Aucune réponse à sa quête d'identité dans l'encyclopédie, pas plus que ne s'y trouve le diagnostic de la maladie qui le ronge. Les marques, les indices glanés ne le rapprochent pas d'une vérité devenue inaccessible avec le décès du père ("pas un des superbes livres [légués par le père] ne répondait à la moindre question que j'aurais voulu poser à l'homme mort ${ }^{2}$ ); ils ne l'éloignent - par le jeu des renvois - pas non plus d'une cause première et indicible (sait-il lui-même ce qu'il cherche?). Alors que la métaphore la plus commune pour qualifier l'encyclopédie a longtemps été l'arbre, l'usage qu'en fait Benoziglio la rapprocherait du rhizome de Deleuze et Guattari :

I. J.-L. Benoziglio, Cabinet portrait, p. 219.

2. Ibid., p. 97. 
pour les désirs, la question n'est jamais de réduire l'inconscient, de l'interpréter ni de le faire signifier suivant un arbre. La question, c'est de produire de l'inconscient, et, avec lui, de nouveaux énoncés, d'autres désirs: le rhizome est cette production d'inconscient même ${ }^{3}$.

\section{Nicolas RIEDER}

Section de français, Faculté des lettres, Université de Lausanne

\section{BIBLIOGRAPHIE}

Barthes, Roland, "Préface au Dictionnaire Hachette» (1980), in

Euvres complètes, t. III, Paris, Seuil, 1995, p. 1227-1229.

Benoziglio, Jean-Luc, Cabinet portrait, Paris, Seuil, 1980.

Deleuze, Gilles, Guattari, Félix, Mille plateaux, Paris, Minuit, 1980.

Demanze, Laurent, Les fictions encyclopédiques, Paris, Corti, 2015.

Mollier, Jean-Yves, Une autre histoire de l'édition française, Paris, La Fabrique, 2015.

Rey, Alain, Miroirs du monde. Une histoire de l'encyclopédisme, Paris, Fayard, 2007.

3. G. Deleuze, F. Guattari, Mille plateaux, p. 27. 